21 Keywords: motor neurons, motor units, electrodes, coherence, connectivity, Renshaw cell,

\section{ACCURATE DECODING OF THE SPINAL CORD OUTPUT IN HUMANS WITH}

$$
\text { IMPLANTED HIGH-DENSITY ELECTRODE ARRAYS }
$$

Silvia Muceli ${ }^{1}$, Wigand Poppendieck ${ }^{2}$, Aleš Holobar ${ }^{3}$, Simon Gandevia ${ }^{4}$, David Liebetanz ${ }^{5}$, Dario Farina ${ }^{6}$

${ }^{1}$ Department of Electrical Engineering, Chalmers University of Technology, Gothenburg, Sweden

${ }^{2}$ Mannheim University of Applied Sciences, Mannheim, Germany

${ }^{3}$ Faculty of Electrical Engineering and Computer Science, University of Maribor, Maribor, Slovenia

${ }^{4}$ Neuroscience Research Australia and University of New South Wales, Randwick, Sydney, New South Wales, Australia

${ }^{5}$ Department of Neurology, University Medical Center Göttingen, Georg-August University, Göttingen, Germany

${ }^{6}$ Department of Bioengineering, Imperial College London, London, United Kingdom

Correspondence to Silvia Muceli (muceli@ chalmers.se) and Dario Farina

(d.farina@imperial.ac.uk) recurrent inhibition 
ABSTRACT

Invasive electromyography opened a new window to explore motoneuron behaviour in vivo.

However, the technique is limited by the small fraction of active motoneurons that can be concurrently detected, precluding a population analysis in natural tasks. Here, we developed a high-density intramuscular electrode for in vivo human recordings along with a fully automatic methodology that could detect the discharges of action potentials of up to 67 concurrently active motoneurons with $99 \%$ accuracy. These data revealed that motoneurons of the same pool receive common synaptic input at frequencies up to $75 \mathrm{~Hz}$ and that late recruited motoneurons inhibit the discharges of those recruited earlier. These results constitute an important step in the population coding analysis of the human motor system in vivo.

\section{INTRODUCTION}

The introduction of intramuscular needles and wires for electromyography (EMG) by Adrian and Bronk (1929) and Basmajian and Stecko (1962) opened a window to explore the neural underpinning of movement control. By recording muscle fibre action potentials, intramuscular EMG reveals the timing of the action potentials discharged by the innervating spinal motoneurons (MN). The analysis of motor units (MUs) from intramuscular EMG decomposition rapidly became the standard approach to study $\mathrm{MN}$ behaviour in vivo in humans and other species (Desmedt, 1973).

Nonetheless, the use of EMG to assess MNs also imposes some constraints. Some intramuscular electrodes are highly selective to detect the electrical activity of a small number of muscle fibres. This makes it easy to identify the discharge times of a few MUs through EMG decomposition, which is conventionally based on spike sorting of action potentials with similar morphology (LeFever and De Luca, 1982). However, the electrode 
selectivity implies that only a small fraction of the hundreds of active MNs can be studied concurrently. To increase the number of sampled MUs, investigators have serially recorded single MU activity. While serial recordings have unravelled patterns of $\mathrm{MN}$ firing, a $\mathrm{MN}$ population analysis is still missing, which limits our understanding of the process of generation of the neural output of the spinal cord. Currently, there is no robust method that provides simultaneous decoding of a large portion of the active MNs in natural tasks.

The identification of large populations of concurrently active MUs is necessary to characterise the synaptic inputs received by MNs. Coherence among spike trains of the homonymous $\mathrm{MN}$ pool reflects the common synaptic input at various frequency bands. A single $\mathrm{MN}$ cannot accurately sample an input with a frequency greater than half its average discharge rate (Lazar and Pnevmatikakis, 2008; Lazar and Tóth, 2004), which is usually in the range $10-40 \mathrm{~Hz}$ (Enoka and Fuglevand, 2001). As a result, sampling by few MNs limits the frequency range at which coherence (and thus common synaptic input) can be observed. However, as the common synaptic input is spread to the whole MN pool (Farina et al., 2014), pooling the spike trains extracted from large populations of MUs allows sampling at higher frequencies.

As a further example, analysis of the output of a population of MNs is also a way to investigate connectivity among MNs, e.g. due to Renshaw inhibition (Eccles et al., 1961; Renshaw, 1941). Renshaw cells receive collateral projections from MN axons and synapse on MNs mediating recurrent inhibition back to the MN pool. However, the distribution of recurrent inhibition throughout the MN pool is unknown in humans (Alvarez, 2019). Most knowledge about recurrent inhibition stems from experiments on anesthetized animal preparations, and direct translation of findings to human studies of intact MNs during natural behaviour is challenging. Again, technological advances for sampling large populations of 
A way to increase the number of concurrently detected MUs in natural tasks uses decomposition of activity recorded with high-density grids of surface electrodes (Holobar et al., 2009). However, surface EMG only detects the activity of superficial MUs (Farina et al., 2010). As an alternative approach to increase the number of sampled MUs, we previously introduced multichannel intramuscular electrodes based on thin-film technology (Farina et al., 2008; Muceli et al., 2015), which provide a large and unbiased sample of MUs from both deep and superficial muscles. These electrodes comprise a linear array of detection points in a flexible wire that can record across the muscle cross-section. Tens of MUs can be concurrently detected with these systems (Muceli et al., 2015). Yet, these systems are limited to only 16 electrode sites and they require partially manual spike sorting. Spike sorting software for multichannel intramuscular EMG indeed currently relies on human oversight to edit the results (McGill et al., 2005).

When increasing the number of recorded signals, the EMG decomposition process must be applied to each recorded EMG channel. With conventional spike sorting, this increases computation time as well as manual editing of the results (Enoka, 2019).

Alternative to spike sorting, blind source separation (BSS) methods can be applied to separate sources (MUs) when a large number of observations (EMG channels) is available (Negro et al., 2016). However, classic BSS limits the maximum number of extracted sources to the number of observations (in practice to less than the observations).

Here, we describe two breakthroughs in the technology to investigate MN behaviour in vivo. First, we designed, manufactured and tested a novel implantable electrode array for human studies with a much greater number of recording sites and higher site density than any previous systems. The novel design allowed the implantation of the array acutely with needles of similar size to those used in conventional concentric needle recording. Second, we used a fully automatic decomposition algorithm (no manual editing) that enabled the 
decoding of the high-density multiunit recordings with accuracy comparable to that achieved by extensive manual editing of each trace by an expert operator. Further, with this new technology, we addressed two fundamental open questions in MN physiology. We found that a $\mathrm{MN}$ pool receives common synaptic input is a frequency range up to $75 \mathrm{~Hz}$, much greater than previously thought. We then analysed the effect of individual MU discharges on the MN population output to determine the connectivity among MNs.

\section{RESULTS}

\section{Intramuscular thin-film electrode array}

We designed and manufactured a high-density intramuscular array with 40 platinum electrodes of area $5257 \mu \mathrm{m}^{2}$ each (Fig. 1 A), linearly distributed over a 2-cm length. Figure 1B shows the complete layout of the double-sided thin-film structure. The structure is built on a polyimide substrate, has a total length of $7 \mathrm{~cm}$ and is U-shaped with two filaments of width $655 \mu \mathrm{m}$ and $150 \mu \mathrm{m}$ (Fig. 1C), and thickness of $20 \mu \mathrm{m}$. The wider filament contains two linear arrays of 20 oval electrodes each (Fig. 1A), with 1-mm inter-electrode distance on the top (cyan) and bottom (green) sides of the polyimide (Fig. 1C). The two arrays have a shift of $0.5 \mathrm{~mm}$ (Fig. 1C). Since the double-sided structure is only $20-\mu \mathrm{m}$ thick, it is equivalent to a linear array of electrodes with $0.5 \mathrm{~mm}$ inter-site distance. The number of electrodes is limited by the number of interconnection lines fitting on the filament. The advantage of two arrays on the two sides of the structure is that the filament width can be reduced for a given number of electrodes. Also, the occurrence of short-circuits during manufacturing is reduced. The narrower filament is inserted into a 25-gauge needle (100 Sterican, B. Braun, Melsungen, Germany), to introduce the thin-film structure into a muscle, with a procedure similar to that used in classic fine wire EMG. The needle is withdrawn leaving the array inside the muscle. 


\section{Signal quality and motor unit yield}

The electrode array was tested in three healthy men (S1-S3). Two arrays were inserted in the tibialis anterior of subject S1, while one array was implanted in the other two subjects. S1 performed a steady contraction at $20 \%$ of maximal force (MVC), whereas S2 and S3 contracted the tibialis at 30\% MVC. The electrodes recorded high quality signals, with a baseline noise of $15.8 \pm 9.9 \mu \mathrm{V}$ (average \pm standard deviation across 4 arrays of 40 channels each). Figure 1D displays representative signals recorded from S1 to show the signal-to-noise ratio. Figure 1E shows the firing patterns of the MUs extracted via manual decomposition from the signals recorded from array1 in S1. In the raster plot, each row represents a different MU, and each vertical line the discharge of an action potential. Within the selected time frame (5 s), 45 MUs were consistently active, $1 \mathrm{MU}$ was recruited during the contraction and 1 had a few isolated discharges. Figure 3F shows a representative example of a MU action potential detected across several electrodes of the 40-channel array. potential trains by two expert investigators ( $\mathrm{SM}$ and $\mathrm{AH}$ ). We refer to the two decomposition processes as manual and automatic decomposition. For manual decomposition, intramuscular EMG signals from each thin-film system were decomposed channel by channel using spike sorting software (McGill et al., 2005), manually edited for resolving missed discharges and superimpositions, and merged (after resolving differences in the discharge patterns of the same MU extracted from different channels) so that each MU's activity was represented by a unique firing pattern. For automatic decomposition, all signals from the same array were decomposed with the BSS method (see Methods and Holobar and Zazula (2007)). We then 
compared the MU firing patterns extracted by the two decomposition procedures (manual and automatic) via the rate of agreement (RoA).

MUs was manually decomposed from the signals recorded from the 4 arrays, yielding 38735 from 0 to $11 \%$, confirming that all identified MU spike trains had few common discharges, i.e., they were unique. The number of channels in which the peak-to-peak amplitude of the corresponding action potential exceeded 10 times the RMS baseline noise ranged from 4 to 40 (median 18) for all MUs but 3 (148 MUs in total). The presence of the same MU over multiple channels contributed to the accurate extraction of the MU firing patterns (Mambrito and De Luca, 1984). The average firing rate was $14.8 \pm 1.7,11.0 \pm 1.2$, and $12.7 \pm 1.9 \mathrm{~Hz}$ for

S1-3, in agreement with previous studies (Connelly et al., 1999; Erim et al., 1996). Most

MUs were active for the whole $20 \mathrm{~s}$ interval, but 10 of 161 fired less than 50 times each and between array1 and array2 of S1 (RoA between all possible pairs (1800) ranged between 0 and 5\%). The cross-spike triggered averaging procedures produced averages at the baseline noise level, further confirming that there were no MUs in common between array 1 and array2.

Decomposition accuracy 

manual and automatic decomposition procedures. About $80 \%$ of the MUs identified by manual decomposition were identified by the automatic decomposition. Only one MU identified by automatic BSS did not match a MU extracted by manual decomposition. The investigator who performed the manual decomposition initially identified the unmatched MU, but she discarded it from further analysis because of lack of confidence in the decomposition accuracy due to the low amplitude of its action potentials. Eight MUs that were not extracted by the automatic decomposition (21\%) fired less than 50 times. procedures (manual and automatic) was $99 \pm 3 \%$. Of those 123 spike trains, 64 matched the automatic results with a $100 \%$ RoA, and 36 had a RoA $\geq 99 \%$. We inspected the disagreement between the output of the two procedures and found that only 3 common MUs had a RoA in the range 80 to $85 \%$ due to misalignments in discharge timings which was greater than our strict threshold of $0.5 \mathrm{~ms}$. One of the three MUs had a satellite action potential. Among the common MUs, 16 discharges identified by the manual decomposition and missed by the automatic decomposition were doublets.

Taken together, these results indicate that the high-density intramuscular array yields high MU sampling and the activity of most of the MUs can be reliably extracted by a fully automatic procedure with comparable accuracy to manual decomposition.

\section{Motor unit population coherence}

We calculated the coherence between groups of MUs of increasing numerosity (Fig. 
statistically significant (i.e., above the $95 \%$ confidence level) for frequencies of about $75 \mathrm{~Hz}$, proving that the synaptic input bandwidth goes well beyond the $\beta$ band. Similarly, the coherence was still significant at $\sim 75 \mathrm{~Hz}$ for S3 (Fig. 2D). In both cases, an increase of coherence in the gamma band with the number of MUs is clear. On the contrary, for S2, the coherence bandwidth was limited to $40 \mathrm{~Hz}$ (Fig. 2C).

\section{[FIGURE 2]}

\section{Reciprocal effect of motoneuron discharges on the homonymous pool}

The discharge of a MN depends on supraspinal and spinal inputs, including from interneurons. A particular class of interneurons, the Renshaw cells, cause recurrent inhibition of the homonymous MN pool (Hultborn et al., 1979). Renshaw cells are facilitated during weak and inhibited during strong contractions (Hultborn and Pierrot - Deseilligny, 1979). We expected to see the effects of reciprocal inhibition in our recordings when the subject exerted forces of 20 or $30 \%$ MVC. As there are opposing views on the distribution of recurrent inhibition between early- and late-recruited MUs within the same MN pool (Granit et al., 1957; Haase et al., 1975; Hultborn et al., 1988), we separately investigated higher and lower threshold MUs. Results are reported in Fig. 3 as synchronization cross-histograms. Firing rate was considered a surrogate of recruitment order, in that early recruited MUs discharge faster, at a given moderate level of force, than those recruited later (De Luca and Erim, 1994). As can be observed in both $\mathrm{S} 1$ and $\mathrm{S} 3$, late recruited MNs caused more inhibition of the discharges of the early recruited MNs at $\sim 15 \mathrm{~ms}$ (dip in Fig. $3 \mathrm{~A}$ and C) than the converse.

On the other hand, for S2 (Fig. 3B), inhibition continued up to $40 \mathrm{~ms}$. No dips were observed in the cross-histograms obtained by applying different perturbations (see METHODS, Connectivity among motoneurons) to the original firing patterns and 
maintaining the firing rate unchanged (control condition; results not shown), implying that the latter did not influence the results presented.

\section{[FIGURE 3]}

\section{DISCUSSION}

We have presented the development of a high-density electrode array for intramuscular recordings that enables the automatic accurate extraction of tens of MUs concurrently active. We have shown representative examples of MU population analysis enabled by our system.

\section{Intramuscular array}

Our electrode array configuration consists of polymer (Hassler et al., 2011) and metal that are micromachined (Stieglitz et al., 2000) into a thread containing 40 electrodes. The materials and minimal thickness $(20 \mu \mathrm{m})$ confer the required flexibility to interface the muscle without being unpleasant for the subject. Each electrode has an area of $5257 \mu \mathrm{m}^{2}$.

Such small electrodes inevitably present high electrical impedance which reduces the signalto-noise ratio. The contacts were therefore coated with microrough platinum that increases the active surface and reduces the impedance by 10 times compared to an untreated electrode (Muceli et al., 2015, 2019). The array has electrodes manufactured on both sides of the substrate (Poppendieck et al., 2015) to enable increased spatial resolution and to reduce the electrodes in a 2-cm long filament.

\section{Motor unit decomposition}

Four intramuscular electrode arrays were tested in 3 subjects. Electrodes were inserted into the tibialis anterior and used to acquire EMG during isometric contractions at moderate 
force. Each array yielded an average number of 40 concurrently active MUs. Eighty-six MUs could be extracted from a contraction at $20 \%$ MVC with two high-density electrode arrays in S1. Given that the tibialis anterior is assumed to comprise about 450 MUs (Enoka, 1995) and the relatively low muscle force exerted by S1, the identified 86 MUs represent a relative large proportion of those that were active during the contraction.

On average, 31 MUs per array could be automatically decomposed with an accuracy of $99 \%$ when compared with manual expert decomposition. Compared to previous systems with fewer electrodes (Muceli et al., 2015), the number of automatically extracted MUs with the proposed high-density electrode is 2 to 3 times greater and the accuracy substantially higher (Negro et al., 2016). For example, our previous attempt at automatic decomposition of EMG recorded with two arrays of 16 channels each yielded 22 out of 53, 24 of out 57, and 21 out of 60 (i.e., about 40\%) manually detected MUs at different force levels, with an average RoA of $94 \%$. Our high-density system enabled automatic decomposition of about $80 \%$ of the manually detected MUs action potential trains constituting the interference EMG with a 99\% RoA. Eight MUs identified by manual decomposition discharged less than 50 times, which was insufficient for the automatic identification. The yield of MUs per channel was also superior to that achieved by BSS of high-density surface EMG data from the tibialis anterior (21 MUs/64 channels) (Del Vecchio et al., 2020) that in any case can only detect MUs with large action potentials at the skin surface.

The automatic decomposition was validated against the manually decomposed dataset. The RoA between the two procedures was 99\% on average (across 123 MUs). This value is remarkably high and can be attributed to the high-density of channels. The comparison between the two decomposition procedures is a conservative approach for estimating accuracy. As signals were decomposed independently by two decomposition methods and operators, the likelihood that the same mistake is made in the two cases is very 
low (Mambrito and De Luca, 1984). Therefore, the procedure of validation of the automatic decomposition in this study is robust. In addition, the average pulse-to-noise ratio across the 124 MUs automatically extracted was $42 \mathrm{~dB}$, greater than values reported for surface EMG decomposition (Holobar et al., 2014), further confirming the high accuracy of the automatic decomposition procedure.

We inspected the disagreement between the two decomposition procedures, and we identified two sources of errors (doublets and misalignments). Some of the doublets could not be identified by the automatic BSS decomposition. This is to be expected as doublets may have an action potential with smaller amplitude compared to the main action potential when the second input (forming the doublet) arrives at the end-plate before the muscle had fully recovered (Denslow, 1948). As the BSS algorithm can only identify action potentials with similar shape, a decrease in amplitude prevented the BSS from associating the doublet to the same MU as the main action potential. Nonetheless, an adaptive change in threshold for detection may in the future solve this problem.

Three MUs found by both decomposition procedures had misalignment for discharges $>0.5 \mathrm{~ms}$ and this influenced the RoA for those MUs. These misalignments are not necessarily errors. The MU action potential train detected at a certain electrode produces time-locked trains in other electrodes that fall in that MU territory, but can also exhibit some jitter from discharge to discharge due to fluctuations in muscle fiber conduction velocity (Stålberg and Sonoo, 1994). In retaining only one firing pattern per MU, we discarded this information on the jitter. Also, one of the three MUs had a satellite potential which showed some size and temporal jitter. The two algorithms may have used either the main potential or the satellite potential as a reference for the alignment, which may then cause misalignments. Note that the results of the automatic decomposition did not undergo any post-processing. Otherwise, some 
mistakes could have been easily corrected by plotting the firing rate against time to detect any inconsistencies.

\section{MU population coherence}

Our coherence analysis showed that the synaptic input common to the MN pool may have frequency content up to $75 \mathrm{~Hz}$ (Fig. $2 \mathrm{~B}$ and D) and that the estimated coherence increases with the number of MUs included in the analysis. Therefore, large populations of concurrently active MUs are necessary to infer characteristics of the neural drive. For a certain frequency of the synaptic input to be detected as common (i.e., statistically significant in the coherence plot), the synaptic input has to be sampled at least twice as fast as that frequency component (Lazar and Pnevmatikakis, 2008). Each MN integrates the supraspinal and afferent inputs and discharges an action potential when the net input exceeds the recruitment threshold. Under the assumption of a common input uniformly distributed to the whole MN pool (Farina et al., 2014), the effective sampling frequency of the synaptic input is the cumulative frequency of all active MNs, i.e. the frequency of the spike train obtained pooling all spike trains together. In voluntary sustained contractions, a MN usually discharges less than 40 action potentials per second (Enoka and Fuglevand, 2001). As a result, sampling by few MNs limits the maximal frequency of the signal recorded from the output of the spinal 
cord, while large populations allow the synaptic input to be reconstructed more accurately from the MN output.

The very large frequency content identified for the neural drive from the spinal cord

(Watanabe and Kohn, 2015). It has long been known that beta oscillations are present in MN output (Ibáñez et al., 2021) while they are filtered out by the muscle contractile properties.

The new observation of a much greater frequency content than the $\beta$ oscillations indicates the variety of common inputs received by the MN pool. Gamma-range cortico-muscular coherence has been observed during strong isometric voluntary contractions (Ushiyama et al., 2012), and during dynamic contractions (Andrykiewicz et al., 2007), suggesting that the gamma-band rhythmic drive from the cortex contributes, at least in part, to the EMG activity at that frequency band. Our results show that human muscles can manifest rhythmic electrical oscillations in the gamma-band also during low intensity isometric contractions.

Reciprocal influence of motoneuron discharges onto the homonymous pool

Our study included the analysis of the influence of the discharges of early recruited MUs on those recruited later (Fig. 3, R1 $\rightarrow$ R2) and vice versa (Fig. 3, R2 $\rightarrow$ R1). We observed that the highest value of the six cross-histograms was obtained at $0 \mathrm{~s}$, indicating the common drive received by the MN pool (De Luca and Erim, 1994). Early recruited MUs were less likely to fire for about $15 \mathrm{~ms}$ (Fig. 3A and C, S1 and S3, R2 $\rightarrow$ R1) or $40 \mathrm{~ms}$ (Fig. 3B, S2, R2 $\rightarrow$ R1) after the discharge of later recruited MUs. This observation fits with recurrent inhibition by Renshaw cells which occurs with similar timing (Bhumbra et al., 
anesthetized animal preparations. The main method to test homonymous recurrent inhibition in humans is indirect and relies on changes in H-reflex modulation caused by presumed recurrent effects (Pierrot-Deseilligny and Burke, 2005). An elegant method to evaluate recurrent inhibition in humans at individual MN level has been proposed by Özyurt et al. (2019). However, this method can only be used to assess the impact of the largest on smaller MUs as it evaluates the effect of electrical stimulation on the background firing of small MUs. On the contrary, our method can be applied in both directions across the MN pool during voluntary contractions. Özyurt et al. (2019) reported an average latency for recurrent inhibition of $37.7 \mathrm{~ms}$ from a peripheral stimulus for the soleus muscle, which is compatible with the dips at $40 \mathrm{~ms}$ visible in the cross-histograms of S2 (Fig. 3B). For S1 and S3, inhibition occurred earlier than for S2 (Fig. 3A and C).

In conclusion, we present a novel high-density intramuscular array along with a methodology that fully automatically identifies the spike trains of relatively large number of

347 MUs, unveiling new knowledge behind MN population coding. We demonstrated that the number of automatically identified MUs is high enough to reveal the presence of significant coherence between groups of MNs in the frequency range up to $75 \mathrm{~Hz}$ and the effect of

350 Renshaw inhibition on the homonymous MN pool. These results constitute an important step

351 forward in the in vivo population coding analysis of the human motor system.

\section{ACKNOWLEDGEMENTS}

354 College London, for useful discussion. 


\section{REFERENCES}

356 Adrian, E.D., and Bronk, D.W. (1929). The discharge of impulses in motor nerve fibres. Part

357 II. The frequency of discharge in reflex and voluntary contractions. J. Physiol. 67, 119-151.

358 Alvarez, F.J. (2019). A motor physiology recurrent topic: simplify assumptions to gain extra 359 insight. J. Physiol. 597, 2117-2118.

360 Andrykiewicz, A., Patino, L., Naranjo, J.R., Witte, M., Hepp-Reymond, M.C., and Kristeva, 361 R. (2007). Corticomuscular synchronization with small and large dynamic force output. BMC Neurosci. 8, 1-12.

Baldissera, F., Cavallari, P., and Cerri, G. (1998). Motoneuronal pre-compensation for the

364 low-pass filter characteristics of muscle. A quantitative appraisal in cat muscle units. J. Physiol. 511, 611-627.

Basmajian, J., and Stecko, G. (1962). A new bipolar electrode for electromyography. J. Appl. Physiol. 17, 849.

Bhumbra, G.S., Bannatyne, B.A., Watanabe, M., Todd, A.J., Maxwell, D.J., and Beato, M. (2014). The recurrent case for the Renshaw cell. J. Neurosci. 34, 12919-12932.

Connelly, D.M., Rice, C.L., Roos, M.R., and Vandervoort, A.A. (1999). Motor unit firing rates and contractile properties in tibialis anterior of young and old men. J. Appl. Physiol. 87, $843-852$.

Denslow, J.S. (1948). Double discharges in human motor units. J. Neurophysiol. 11, 209215. 
377 Eccles, J.C., Eccles, R.M., Iggo, A., and Lundberg, A. (1961). Electrophysiological investigation of Renshaw cells. Electrophysiol. Investig. Renshaw Cells 159, 461-478.

Enoka, R.M. (1995). Morphological features and activation patterns of motor units. J. Clin. Neurophysiol. 12, 538-559.

Enoka, R.M. (2019). Physiological validation of the decomposition of surface EMG signals.

Enoka, R.M., and Fuglevand, A.J. (2001). Motor unit physiology: some unresolved issues.

Erim, Z., De Luca, C.J., Mineo, K., and Aoki, T. (1996). Rank-ordered regulation of motor units. Muscle and Nerve 19, 563-573.

Farina, D., Arendt-Nielsen, L., Merletti, R., and Graven-Nielsen, T. (2002). Assessment of single motor unit conduction velocity during sustained contractions of the tibialis anterior muscle with advanced spike triggered averaging. J. Neurosci. Methods 115, 1-12.

Farina, D., Yoshida, K., Stieglitz, T., and Koch, K.P. (2008). Multichannel thin-film electrode for intramuscular electromyographic recordings. J. Appl. Physiol. 104, 821-827. muscles from the surface electromyogram. Clin. Neurophysiol. 121, 1616-1623.

Farina, D., Negro, F., and Dideriksen, J.L. (2014). The effective neural drive to muscles is the common synaptic input to motor neurons. J. Physiol. 592, 3427-3441.

396 Granit, R., Pascoe, J.E., and Steg, G. (1957). The behaviour of tonic $\alpha$ and $\beta$ motoneurones 397 during stimulation of recurrent collaterals. J. Physiol. 138, 381-400. 
399

400

401

402

403

404

405

406

407

408

409

410

411

412

413

414

415

416

417

418

419

420

recurrent inhibition in the mammalian spinal cord. Rev. Physiol. Biochem. Pharmacol. 73, 73-129.

Hassler, C., Boretius, T., and Stieglitz, T. (2011). Polymers for neural implants. J. Polym. Sci. Part B Polym. Phys. 49, 18-33.

Holobar, A., and Zazula, D. (2007). Multichannel blind source separation using convolution kernel compensation. IEEE Trans. Signal Process. 55, 4487-4496.

Holobar, A., Farina, D., Gazzoni, M., Merletti, R., and Zazula, D. (2009). Estimating motor unit discharge patterns from high-density surface electromyogram. Clin. Neurophysiol. 120, $551-562$.

Holobar, A., Minetto, M.A., Botter, A., Negro, F., and Farina, D. (2010). Experimental analysis of accuracy in the identification of motor unit spike trains. IEEE Trans. Neural Syst. Rehabil. Eng. 18, 221-229.

Holobar, A., Minetto, M.A., and Farina, D. (2014). Accurate identification of motor unit discharge patterns from high-density surface EMG and validation with a novel signal-based performance metric. J. Neural Eng. 11, 016008.

Hultborn, H., and Pierrot-Deseilligny, E. (1979). Changes in recurrent inhibition during voluntary soleus contractions in man studied by an H-reflex technique. J. Physiol. 297, 229_ 251.

Hultborn, H., Lindström, S., and Wigström, H. (1979). On the function of recurrent inhibition in the spinal cord. Exp. Brain Res. 37, 399-403.

Hultborn, H., Katz, R., and Mackel, R. (1988). Distribution of recurrent inhibition within a motor nucleus. II. Amount of recurrent inhibition in motoneurones to fast and slow units. 
Acta Physiol. Scand. 134, 363-374.

422 Ibáñez, J., Del Vecchio, A., Rothwell, J.C., Baker, S.N., and Farina, D. (2021). Only the

423 fastest corticospinal fibers contribute to $\beta$ corticomuscular coherence. J. Neurosci. 41, 48674244879.

Lazar, A.A., and Pnevmatikakis, E.A. (2008). Faithful representation of stimuli with a population of integrate-and-fire neurons. Neural Comput. 20, 2715-2744.

Lazar, A.A., and Tóth, L.T. (2004). Perfect recovery and sensitivity analysis of time encoded bandlimited signals. IEEE Trans. Circuits Syst. I Regul. Pap. 51, 2060-2073.

LeFever, R.S., and De Luca, C.J. (1982). A procedure for decomposing the myoelectric signal into its constituent action potentials - Part I: technique, theory, and implementation. IEEE Trans. Biomed. Eng. BME-29, 149-157.

De Luca, C.J., and Erim, Z. (1994). Common drive of motor units in regulation of muscle force. Trends Neurosci. 17, 299-305.

Mambrito, B., and De Luca, C.J. (1984). A technique for the detection, decomposition and analysis of the EMG signal. Electroencephalogr. Clin. Neurophysiol. 58, 175-188. decomposition program. J. Neurosci. Methods 149, 121-133. S.C., and Farina, D. (2015). Accurate and representative decoding of the neural drive to muscles in humans with multi-channel intramuscular thin-film electrodes. J. Physiol. 593, 3789-3804. 
Pons, J.L., and Farina, D. (2019). A thin-film multichannel electrode for muscle recording

Negro, F., Muceli, S., Castronovo, A.M., Holobar, A., and Farina, D. (2016). Multi-channel

Özyurt, M.G., Piotrkiewicz, M., Topkara, B., Weisskircher, H.W., and Türker, K.S. (2019).

Motor units as tools to evaluate profile of human Renshaw inhibition. J. Physiol. 597, 21852199.

Pierrot-Deseilligny, E., and Burke, D. (2005). Recurrent inhibition. In The Circuitry of the Microdevices 16, 837-850. K.P. (2015). A new generation of double-sided intramuscular electrodes for multi-channel recording and stimulation. Proc. Annu. Int. Conf. IEEE Eng. Med. Biol. Soc. EMBS 2015Novem, 7135-7138.

Renshaw, B. (1941). Influence of discharge of motoneurons upon excitation of neighboring motoneurons. J. Neurophysiol. 4, 167-183. unit action potential, the "jiggle," at consecutive discharges. Muscle Nerve 17, 1135-1144. 
465 Stieglitz, T., Beutel, H., Schuettler, M., and Meyer, J.-U. (2000). Micromachined, polyimide-

466 based devices for flexible neural interfaces. Biomed. Microdevices 2, 283-294.

467 Ushiyama, J., Masakado, Y., Fujiwara, T., Tsuji, T., Hase, K., Kimura, A., Liu, M., and

468 Ushiba, J. (2012). Contraction level-related modulation of corticomuscular coherence differs

469 between the tibialis anterior and soleus muscles in humans. J. Appl. Physiol. 112, 1258-1267.

470 Del Vecchio, A., Holobar, A., Falla, D., Felici, F., Enoka, R.M., and Farina, D. (2020).

471 Tutorial: Analysis of motor unit discharge characteristics from high-density surface EMG

472 signals. J. Electromyogr. Kinesiol. 53, 102426.

473 Watanabe, R.N., and Kohn, A.F. (2015). Fast oscillatory commands from the motor cortex

474 can be decoded by the spinal cord for force control. J Neurosci 35, 13687-13697. 
FIGURE CAPTIONS

FIGURE 1: Design of the double-sided electrode array and representative recordings. (A)

477 Close-up of an oval electrode. (B) Whole structures with the tracks running towards the

478 connection pad. (C) Close-up of the electrode array tip. Electrodes represented in cyan are

479 located on the top side of the thin-film array and those in green are located on the bottom side

480 of the wider filament. (D) Representative recordings obtained from the tibialis anterior of S1

481 during a contraction at $20 \%$ of the maximal force (MVC). (E) Firing pattern of 45 MUs

extracted from the signal shown in D. (F) Multichannel action potentials of a representative motor unit obtained by averaging the red-coloured EMG channels in panel D with the firing pattern of the same colour in panel $\mathrm{E}$ as a trigger.

FIGURE 2. Coherence between populations of motor units. (A) Firing pattern of 68 motor units active during $20 \mathrm{MVC}$ contraction (S2, 2 arrays). Coherence between combinations of cumulative spike trains (CSTs) obtained by pooling an increasing number of motor units from subject S1 (B), S2 (C), and S3 (D). Black dashed horizontal line is the $95 \%$ confidence limit. Coherence increased with the motor unit numerosity and the population coherence was significant up to $40 \mathrm{~Hz}$ in S2, and up to $75 \mathrm{~Hz}$ in S1 and S3, respectively. Note: $60 \mathrm{~s}$ of data were used for S1, $20 \mathrm{~s}$ for $\mathrm{S} 2$ and $\mathrm{S} 3$.

FIGURE 3. Analysis of motor unit synchronization for subjects S1 (A), S2 (B), and S3 (C).

Left panels show the average discharge rate of the motor units in a $20 \mathrm{~s}$ time interval. Central

$494 \mathrm{R} 1 \rightarrow \mathrm{R} 2(\mathrm{R} 2 \rightarrow \mathrm{R} 1)$ panels display the influence of earlier (later) recruited motor units on the discharge timing of the later (earlier) recruited motor units via cross-histograms between pairs of motor unit spike trains. The two rightmost columns represent the same values in logarithmic scale so that the inhibition can be more readily visualised. 
498 TABLE 1: Decomposition performance for the high-density intramuscular signals: manual

499 versus automatic decomposition

\begin{tabular}{|c|c|c|c|c|c|}
\hline ID & $\begin{array}{c}\text { Number of } \\
\text { MUs } \\
\text { (manual) }\end{array}$ & $\begin{array}{c}\text { Number of } \\
\text { MUs } \\
\text { (automatic) }\end{array}$ & $\begin{array}{c}\text { Number of } \\
\text { MUs } \\
\text { (common) }\end{array}$ & $\begin{array}{c}\text { RoA } \\
\text { (mean } \pm \text { SD, }\end{array}$ & $\begin{array}{c}\text { PNR } \\
\text { (automatic, } \\
\text { dB) }\end{array}$ \\
\hline $\begin{array}{c}\text { S1 } \\
\text { array1 }\end{array}$ & 50 & 40 & 39 & $99 \pm 3$ & $40.5 \pm 7.4$ \\
\hline $\begin{array}{c}\text { S1 } \\
\text { array2 }\end{array}$ & 36 & 27 & 27 & $98 \pm 4$ & $41.1 \pm 6.7$ \\
\hline S2 & 39 & 27 & 27 & $100 \pm 1$ & $42.0 \pm 5.3$ \\
\hline S3 & 36 & 30 & 30 & $99 \pm 4$ & $44.9 \pm 8.5$ \\
\hline
\end{tabular}

500 MU: motor unit; RoA: rate of agreement; SD: standard deviation; PNR: pulse to noise ratio 
METHODS

502

\section{Manufacturing process}

The thin-film electrode array structure was built using microfabrication processes.

The electrode array was built over a silicon wafer used as a platform for the production. The structure was built layer by layer with layers of metal for tracks sandwiched between three layers of polyimide. Metals were patterned using a photolithography process.

First, a platinum etch mask was deposited and lift-off structured on a 4 inches silicon wafer. In the next step, a $5 \mu \mathrm{m}$ polyimide layer (PI 2611, HD Microsystems) was spun on the wafer and cured at $350^{\circ} \mathrm{C}$. The lower platinum electrode contacts and tracks were then sputtered and lift-off structured. Another $10 \mu \mathrm{m}$ polyimide layer was deposited, followed by the upper platinum electrode tracks and contacts, which were sputtered and lift-off structured, followed by a final $5 \mu \mathrm{m}$ polyimide layer for insulation. To reach the contacts on the lower side, the silicon wafer was etched from the backside using reactive ion etching. In a second reactive ion etching step, the lower electrode contacts were opened using the previously deposited platinum layer as etch mask. An aluminum etch mask was then deposited on the top side and used for reactive ion etching of the polyimide to open the contacts on the upper side. After removal of the aluminum mask, the microfabrication process was completed, and the separated double-sided electrode arrays were removed from the wafer using tweezers.

The electrode contacts were coated with microrough platinum using electroplating from an aqueous solution of hexachloroplatinic acid (Poppendieck et al., 2014). This reduced the electrode impedance by about one order of magnitude so that the resulting values of impedance spectroscopy were $\sim 10 \mathrm{k} \Omega$ at $1 \mathrm{kHz}$. A plug (Harwin M50-4902045 connector) was soldered to the adapter as the interface with external hardware. Each electrode array was 
inserted into a hypodermic needle with the bevel smoothed with a laser (PICCO LASER,

525 O.R. Lasertechnologie, DE).

Three healthy men (age range: 29 - 39 years) participated in the experiment, which was approved by the Ethical Committee of the University Medical Center of Göttingen and conducted according to the Declaration of Helsinki (2008).

\section{Experimental procedure}

Inc., NY, USA) with the right leg and foot stably fixated. He was asked to perform two brief maximal voluntary contractions with 5 minutes interval in between to recover from fatigue.

The peak of the two was considered as the maximal voluntary contraction (MVC). Electrode array placement followed 5 extra minutes of rest. The skin was cleaned with alcohol and the thin-film electrode array(s) were inserted into the middle of the proximal half of the tibialis anterior muscle, perpendicular to the skin with the tip of the needle to a depth of $2.5 \mathrm{~cm}$ below the fat layer as estimated by ultrasound (Telemed Ltd. Vilnius, Lithuania). The two electrode arrays in $\mathrm{S} 1$ were about 3 and $1 \mathrm{~cm}$ distant in the longitudinal and perpendicular direction of the muscle, respectively. USB2, OT-Bioelettronica, Torino, Italy) with a gain of 200-500, and band-pass filtered (8th order Bessel filter, high-pass cut-off frequency 10-100; low-pass cut-off frequency $4400 \mathrm{~Hz}$ ),

544 before being sampled at $10 \mathrm{kHz}$, using a 12-bit A/D converter. The EMG signals were acquired in a unipolar derivation with reference and ground electrodes at the ankle. 
547 which the experimenters judged the signal quality. Following these trials, S1 was asked to perform a steady contraction at 20\% MVC, whereas S2 and S3 were given $30 \%$ MVC as the target force level. Subjects were asked to perform a steady contraction lasting at least $1 \mathrm{~min}$. The subject was provided with real-time force feedback displayed on a screen. The target force level was represented as straight line on the computer screen and the force exerted by the subject as a running dot. The subject was instructed to keep the position of the dot as close as possible to the straight line. He was allowed to complete the 1 min contraction at once or in multiple contractions with rest at will in between. channels / array) of the root-mean-square of a 4 s segment of data recorded at rest. into the constituent MU action potential trains by two expert investigators (SM and $\mathrm{AH}$, respectively). In both cases, signals were high pass filtered at $250 \mathrm{~Hz}$ prior decomposition. In case of manual decomposition, intramuscular EMG signals from each thin-film array were decomposed using the decomposition software EMGLAB (McGill et al., 2005), that relies on spike sorting to detect MU action potentials. Each channel was decomposed independently and the series of discharges of a single MU were manually edited for resolving missed 
570

571

572

573

574

575

576

577

578

579

580

581

582

583

584

585

586

587

588

589

590

591

potentials from the raw signal, was comparable in power with the raw signal baseline noise, indicating that all MU activity had been accounted for. As the same MU could be detected in adjacent channels, the decomposition results from all channels were then merged by automatically identifying the MUs detected at more than one electrode. Discharge patterns with more than $75 \%$ discharges closer than $1 \mathrm{~ms}$ were considered to belong to the same MU identified on different channels. Differences in the discharge patterns of the same MU extracted from different channels were examined and resolved by the investigator in charge, so that at the final stage of the manual decomposition, each MU was represented by a unique firing pattern.

A second investigator $(\mathrm{AH})$, automatically decomposed the 20 s signals using the convolution kernel compensation algorithm (CKC) (Holobar and Zazula, 2007). To briefly summarize the algorithm working principle, assuming absence of noise, we can express the intramuscular EMG signal $x_{c}(k)$ recorded at channel $c$ as the sum of trains of action potentials (one train for each active MU):

$$
x_{c}(k)=\sum_{i=1}^{M} \sum_{l=0}^{L-1} h_{c i}(l) \sum_{r} \delta\left(k-\phi_{i r}-l\right), k=0, \ldots, f_{S} T
$$

where $f_{S}$ is the sampling frequency, $T$ the signal duration, $h_{c i}(l)$ is the action potential of the $i$-th MU as recorded at the $c$-th channel, $\sum_{r} \delta\left(k-\phi_{\text {ir }}\right)$ the spike train of the $i$-th MU with spikes at times $\phi_{i r}, L$ the duration of the action potentials, and $M$ the number of active MUs.

Equation 1 can be re-written in matrix form as follows:

$$
\underline{x}(k)=\sum_{l=0}^{L-1} \underline{H}(l) \underline{s}(k-l) \quad \text { with } s_{i}(k)=\sum_{r} \delta\left(k-\phi_{i r}\right) .
$$

Once the mixing matrix $\underline{H}$ is identified, the source pulse trains can be extracted by multiplying the EMG signals $(\underline{x})$ by the inverse of $\underline{H}$ (unmixing matrix). The reliability of the 
592

593

594

595

596

597

598

599

600

601

602

603

604

605

606

607

608

609

610

611

612

613

614

615

automatic decomposition was assessed by the pulse-to-noise ratio, which is a signal-based

metric that has been validated to assess the decomposition accuracy of BSS-based

decomposition algorithms (Holobar et al., 2014).

\section{Assessment of the decomposition accuracy}

For each electrode array (3 subjects, 4 arrays), we report the number of MUs identified by the manual and automatic decomposition, and those commonly identified by both approaches.

We first inspected the results of the manual decomposition. We calculated the RoA (Holobar et al., 2010) between each pair of MU firing patterns identified from the same 40 channel array, to ensure that they were unique. The RoA was defined as the ratio between the number of discharges that were present in both firing patterns (common) and the sum of the number of common discharges and the number of discharges present in only one of the two firing patterns. A tolerance of 10 sample $(<1 \mathrm{~ms})$ was used when identifying common discharges.

Each MU firing pattern was accurately estimated from the comparison between the firing patterns of that MU in multiple channels. To assess the robustness of the estimation, we calculated the multichannel MU action potentials by spike triggered averaging (Farina et al., 2002), i.e., by averaging the EMG of each channel using the discharges obtained from decomposition as a trigger. For each MU, we then counted the number of channels where the peak-to-peak amplitude of the action potential was greater than 10 times the average RMS of the baseline noise across the 40 channels. The higher the number of channels exceeding the threshold, the higher the likelihood that the firing pattern was accurately estimated (Mambrito and De Luca, 1984).

The RoA was also used to check whether there were MUs in common between array 1 and array 2 of S1. As a further check, we performed cross-spike triggered averaging by averaging the EMG of each channel of array1 (array2) using the discharges obtained from 
617 (centered about the MU firing) was used in the spike triggered averaging procedure to account for the propagation delay between the position of the electrode arrays, which were about $3 \mathrm{~cm}$ apart. For MUs in common between the two arrays, the cross-averaging procedure will yield an action potential with higher amplitude than the baseline noise. procedures (manual and automatic). Here RoA was defined as the ratio between the matched discharges resulting from the comparison of the two procedures and the sum of matched and unmatched discharges. Discharge patterns with more than $75 \%$ discharges closer than $0.5 \mathrm{~ms}$ were considered to belong to the same MU identified by the manual and automatic procedure (common MU).

\section{MU population coherence}

The discriminated spike trains were used to compute spectral coherence between groups of MUs, with numerosity ranging from 1 to half of the maximum number of extracted MUs. The spike trains) and the average coherence across the 25 repetitions was calculated. For each occurrence of a discharge. Within each MU group, the spike trains were summed to provide a cumulative spike train. Coherence analysis was performed on $0.5 \mathrm{~s}$ non-overlapping Hanning the sampling rate. To define the significance threshold for coherence peaks, the confidence

637 level CL was calculated as (Rosenberg et al. 1989):

$$
C L=1-(1-\alpha)^{\frac{1}{N-1}}
$$


640

641

642

where $N$ and $\alpha$ represent the number of segments used in the coherence calculation (data length/number of windows) and the confidence level (95\%), respectively.

\section{Connectivity among motoneurons}

Connectivity among MNs was estimated by the cross-histogram of the discharge of pairs of MUs (1 ms resolution). To consider the opposing views on the distribution of recurrent inhibition between early- and late-recruited MUs within the homonymous MN pool (Granit et al., 1957; Haase et al., 1975; Hultborn et al., 1988), we investigated separately higher and lower threshold MUs. MUs were ordered by firing rate based on the fact that at a given force, earlier recruited MUs discharge faster than later recruited ones (De Luca and Erim, 1994). As control conditions, we generated 4 types of firing patterns with the same number of discharges as the detected MUs in the same time interval and $i$ ) uniformly distributed discharge times, $i$ ) equal inter-spike intervals, $i i i)$ discharged times obtained from the experimental ones by applying a time shift of 0 to $70 \mathrm{~ms}$ to the whole MU action potential train (different for the different MUs, but the same for all action potentials of the same MU), and $i v$ ) discharged times obtained from the experimental ones by adding or subtracting a time in the range of 0 to $10 \%$ of the average inter-spike interval for each MU (different time shifts for each individual action potential). 


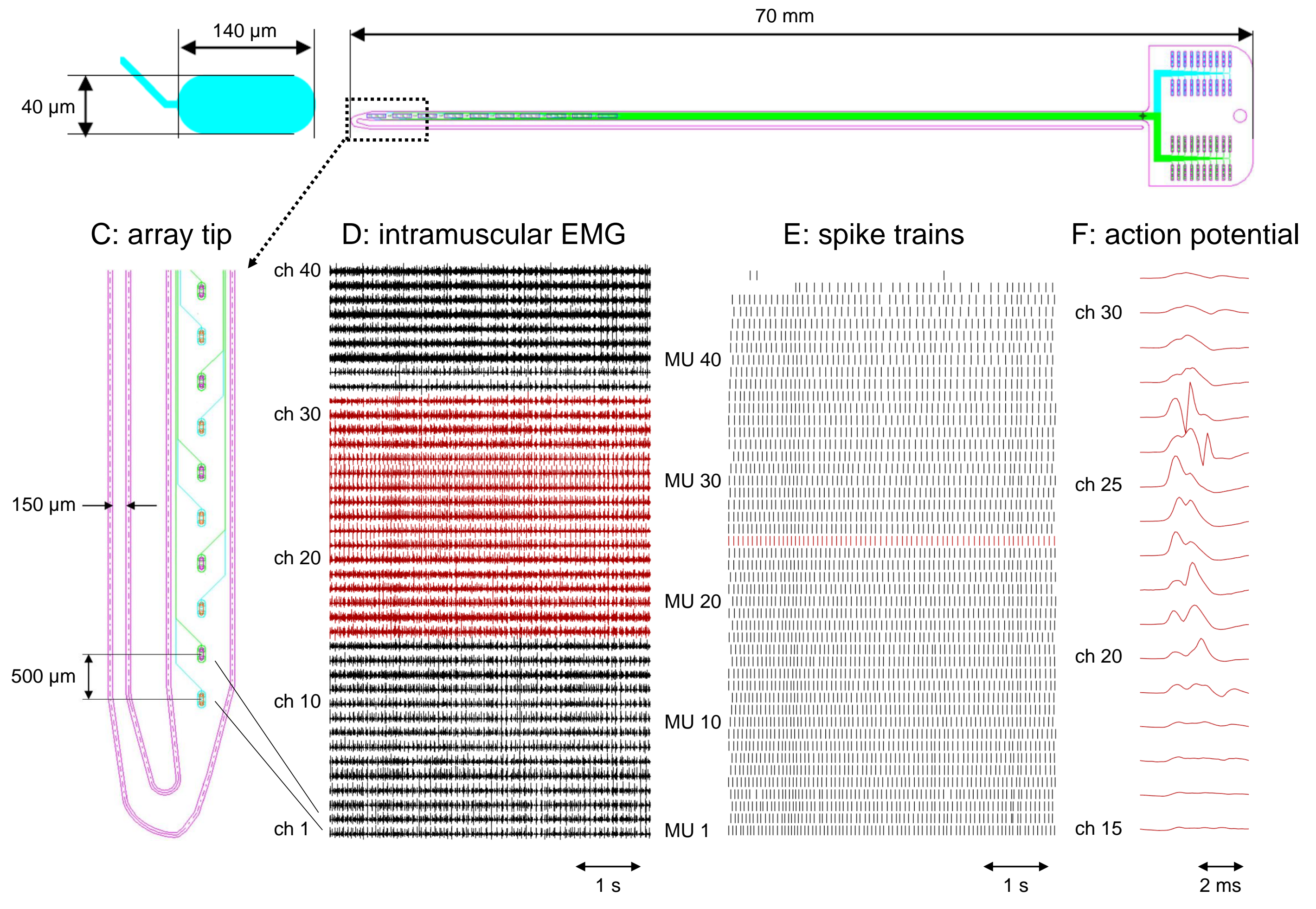


A $\quad 68$

S1

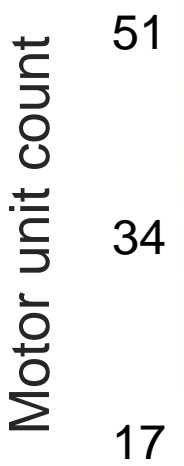

$\mathrm{B}$

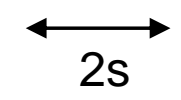

S1

bioRxit preprint doi: https://doi.org/10.1101/2022.01.29.478247; this version posted January 30, 2022. The copyright holder for this preprint (which was not certified by peer review) is the author/funder. All rights reserved. No reuse allowed without permission. 1

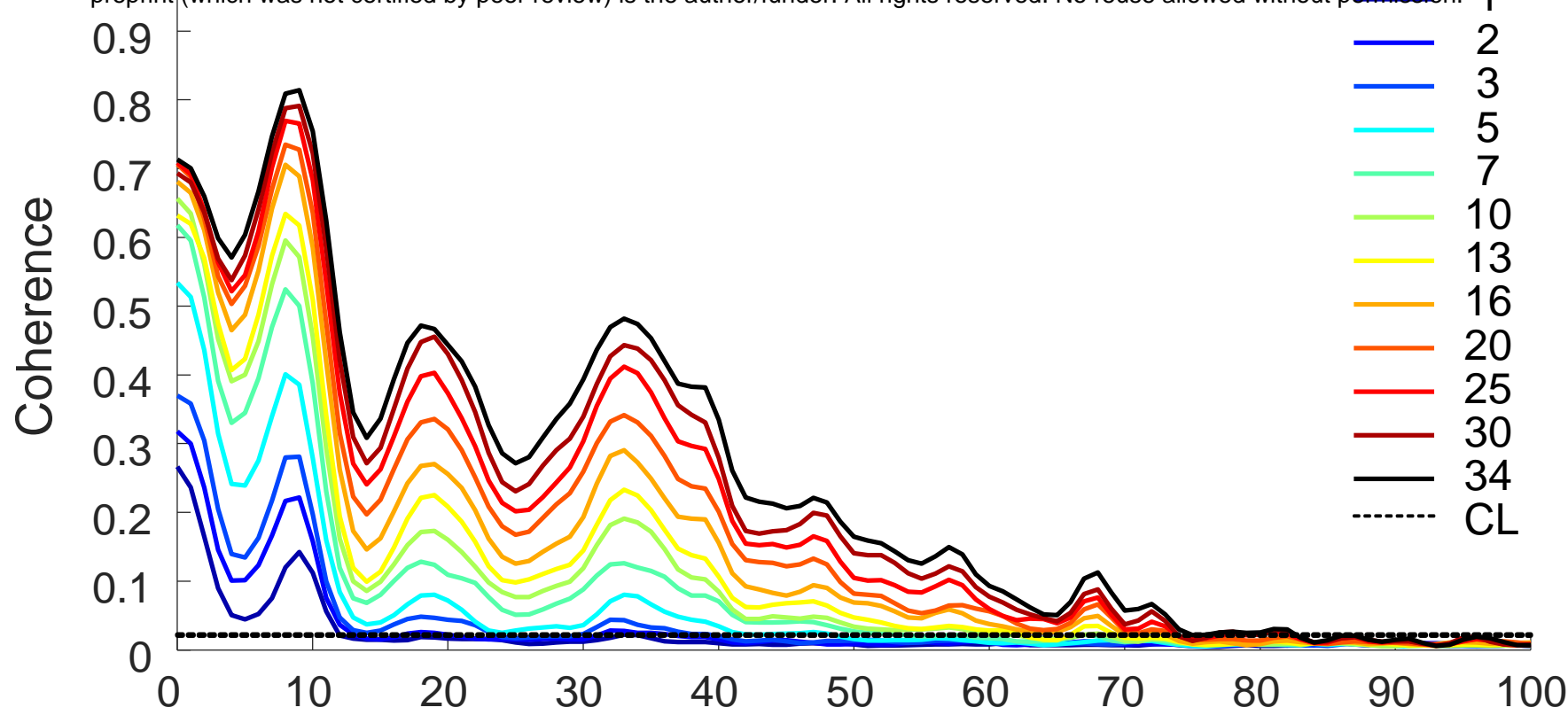

C

Frequency $(\mathrm{Hz})$

S2

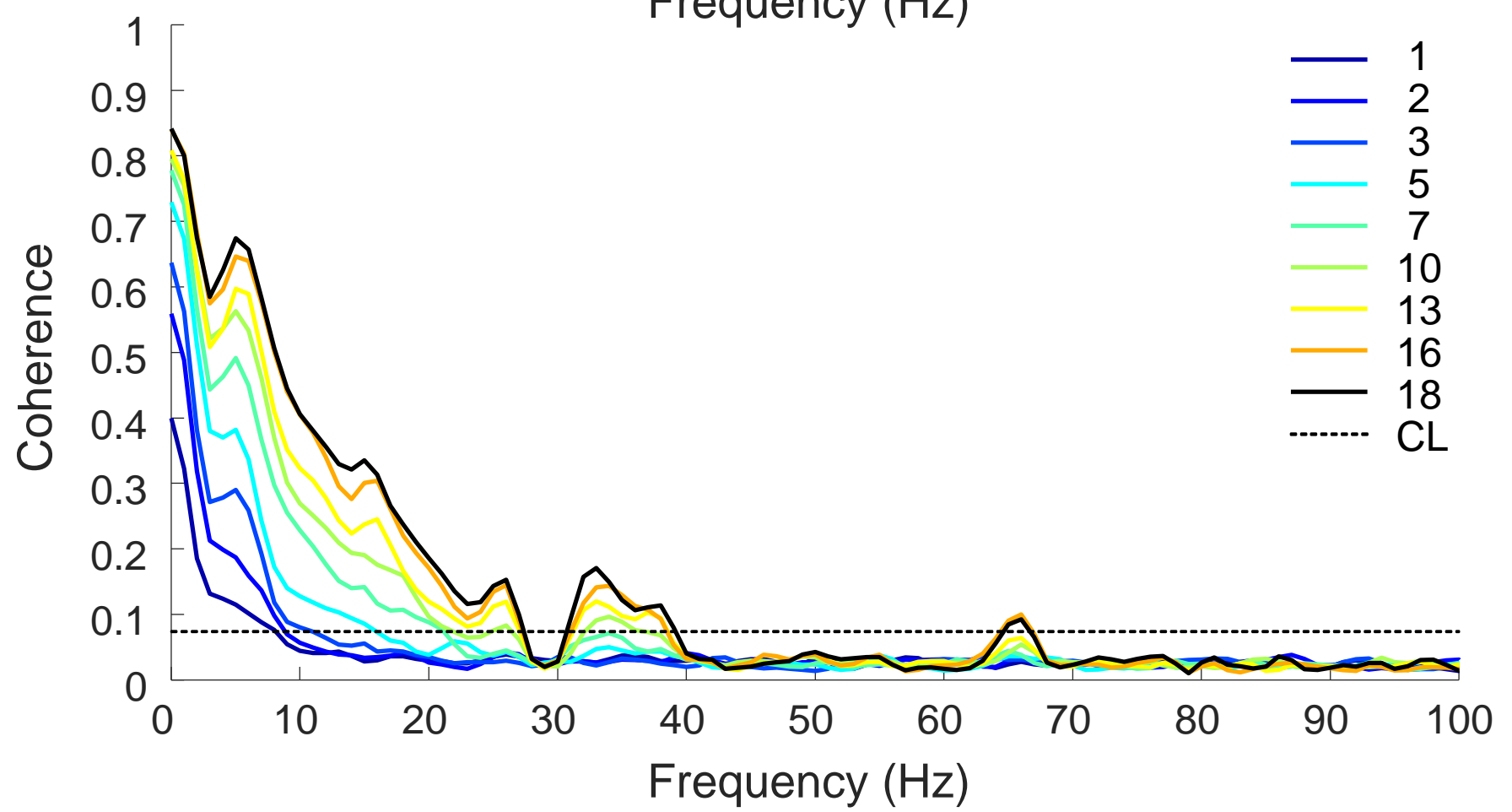

$\mathrm{D}$

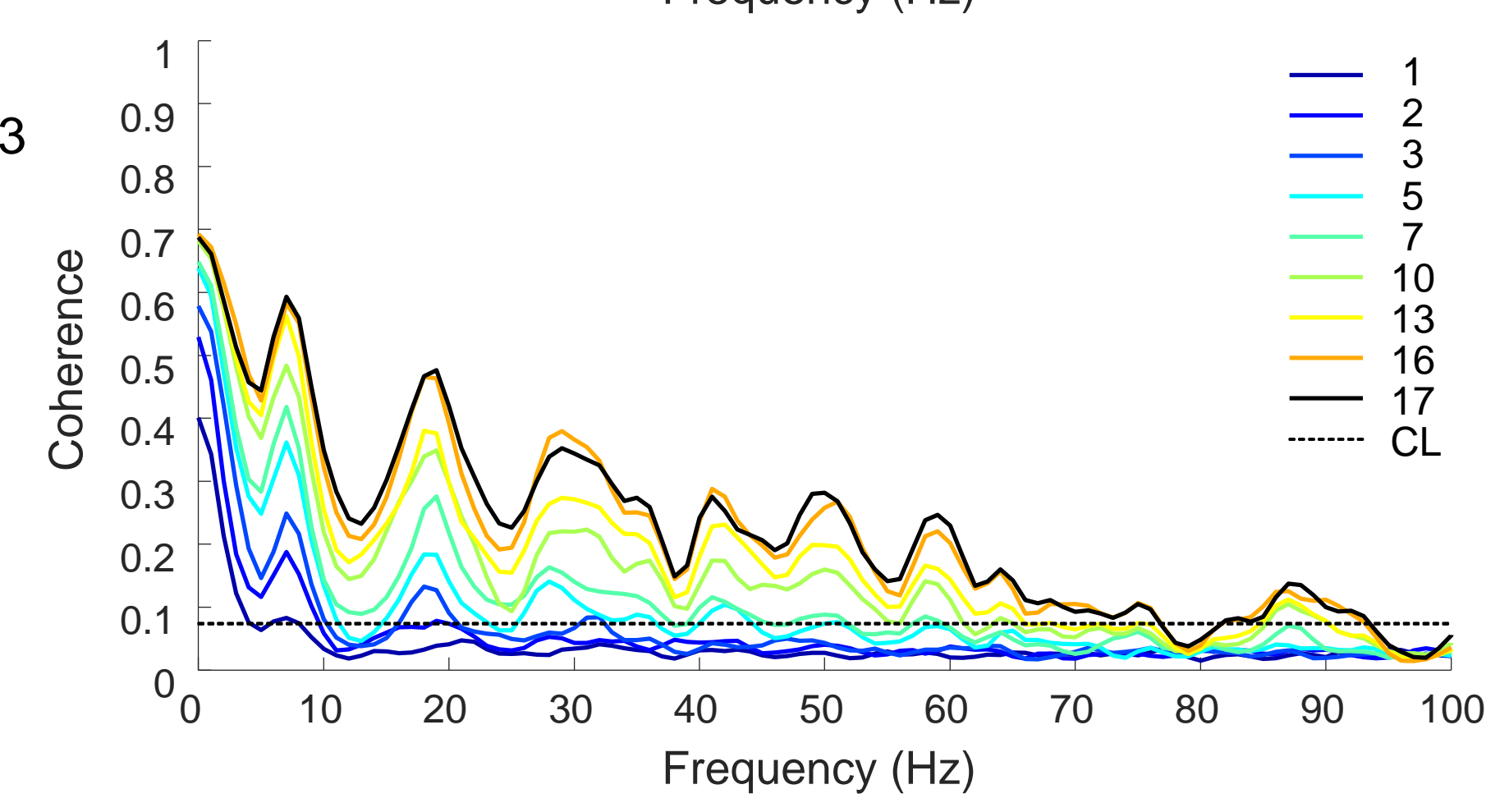

Fig 2 
A S1

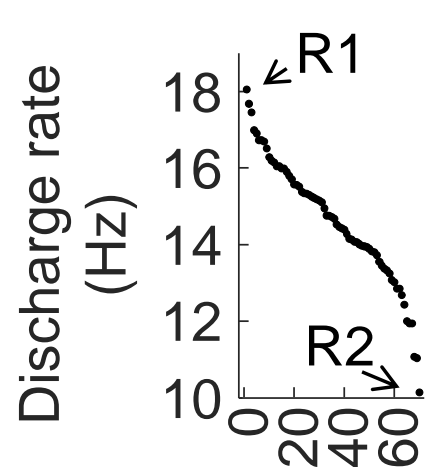

B

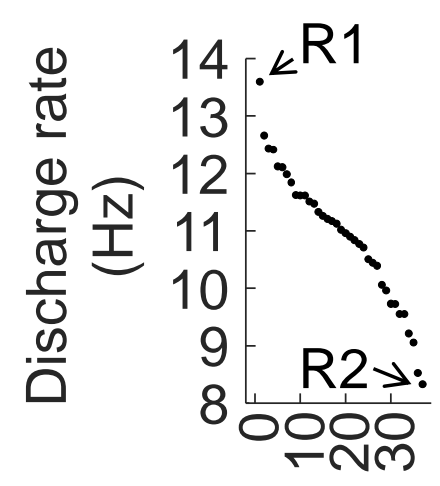

C

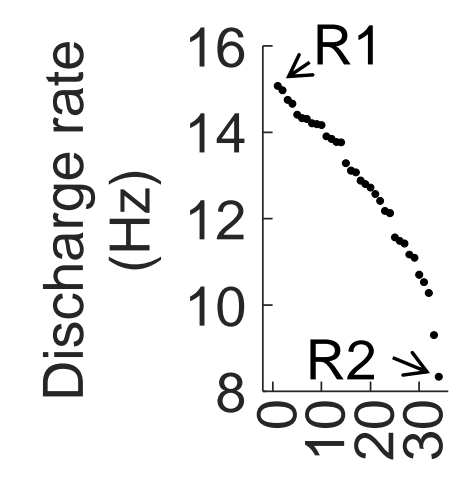

MU number
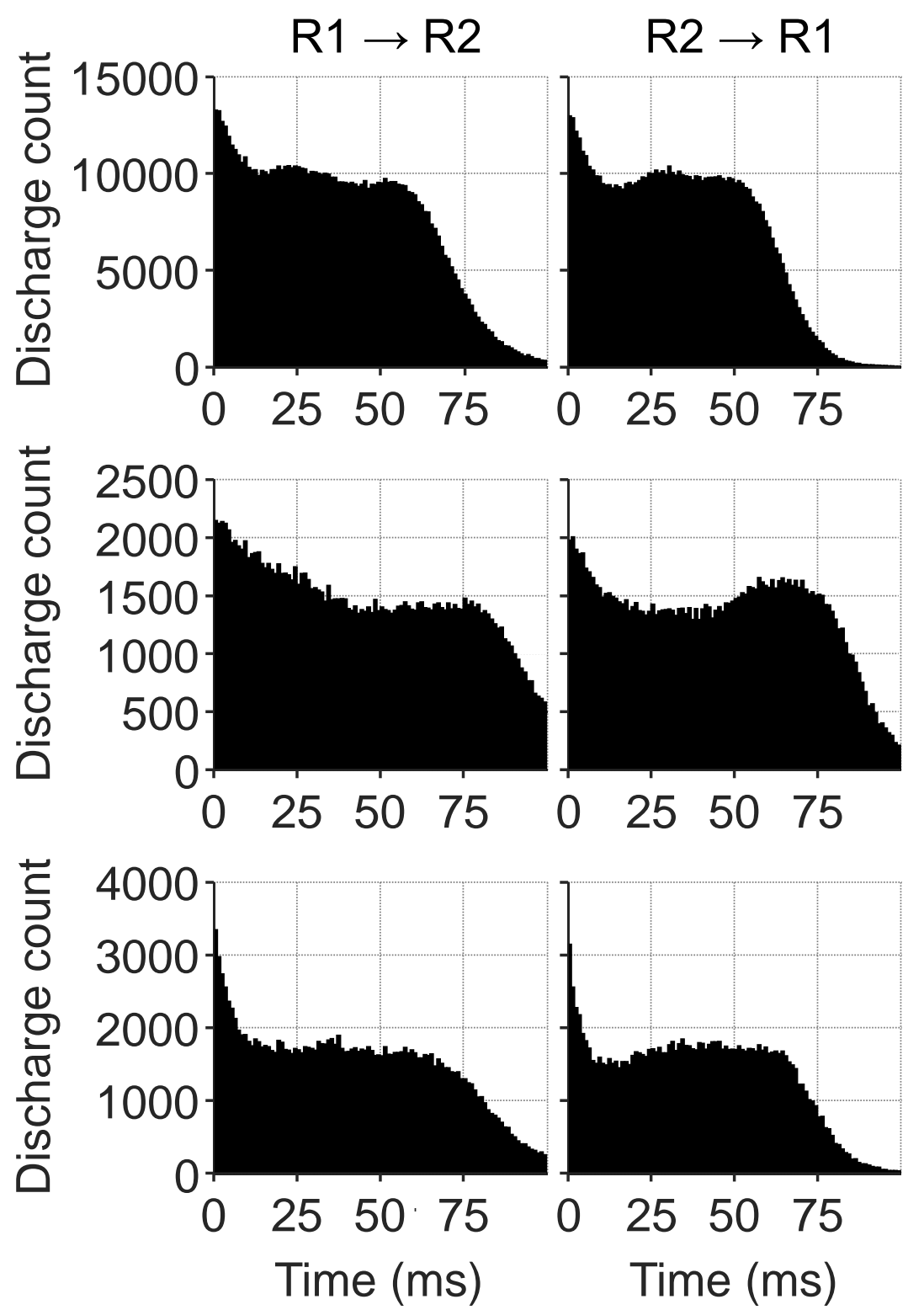
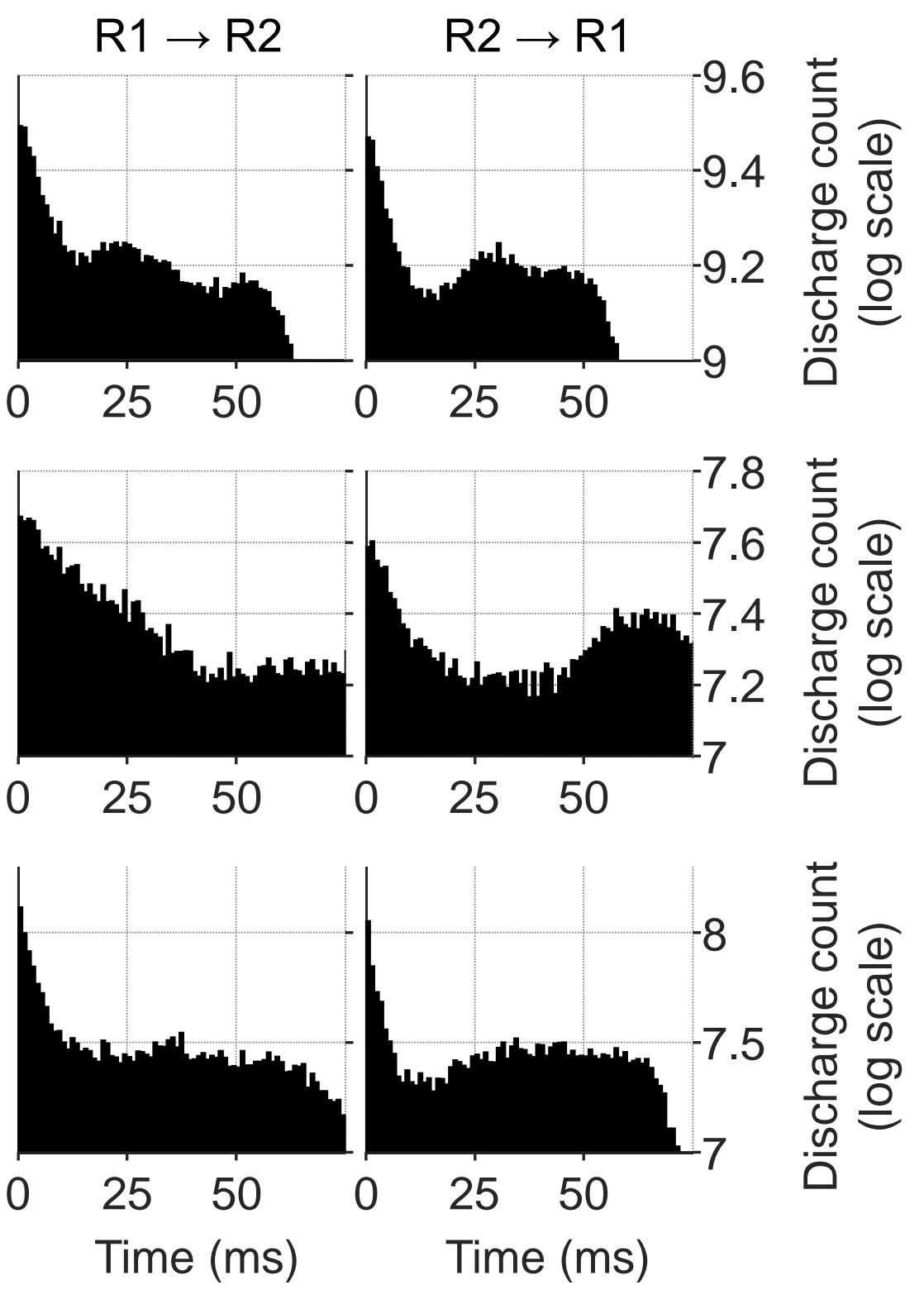

Fig 3 